\section{International Society of Blood Transfusion}

\section{K. Kleesiek ${ }^{1}$, C. Götting ${ }^{2}$, J. Diekmann ${ }^{3}$, J. Dreier ${ }^{4}$ und} M. Schmidt ${ }^{5}$

${ }^{1}$ Ehemaliger Direktor des Instituts für Laboratoriums- und Transfusionsmedizin, Herz- und Diabeteszentrum NordrheinWestfalen Ruhr-Universität Bochum, Bad Oeynhausen,

Deutschland

${ }^{2}$ MVZ Labor Limbach Nürnberg GmbH, Nürnberg, Deutschland

${ }^{3}$ Institut für Laboratoriums- und Transfusionsmedizin, Herzund Diabeteszentrum NRW, Bad Oeynhausen, Deutschland ${ }^{4}$ Herz- und Diabeteszentrum Nordrhein-Westfalen; Institut für Laboratoriums- und Transfusionsmedizin, Universitätsklinik der Ruhr-Universität Bochum, Bad Oeynhausen, Deutschland
${ }^{5}$ Institut für Laboratoriums- und Transfusionsmedizin, Universitätsklinik der Ruhr-Universität Bochum, Bad Oeynhausen, Deutschland

Synonym(e) ISBT; Société Internationale de Transfusion Sanguine (SITS)

Definition Im Jahr 1935 gegründete internationale wissenschaftliche Gesellschaft, die sich zum Ziel gesetzt hat, Studien zur Bluttransfusion zu unterstützen und die Transfusionsmedizin zu fördern. Die Gesellschaft fördert die Standardisierung und Harmonisierung im Bereich der Bluttransfusion. Es wurde dabei u. a. eine Klassifikation der verschiedenen humanen $>$ Blutgruppensysteme unter einer allgemeinen Nomenklatur erarbeitet. 